\title{
Reducing the total ischaemic time in ST-segment elevation myocardial infarction: Every step matters
}

Yew Woon $\underline{\text { Chia }}{ }^{1}{ }_{F R C P}$, Michael Yih Chong $\underline{\text { Chia }}{ }^{2}{ }_{\text {FRCEM }}$

Ischaemic heart disease (IHD) is the third leading cause of death in Singapore, accounting for $18.8 \%$ of all mortalities in $2019 .{ }^{1}$ With our ageing population, there is an increased prevalence of cardiovascular risk factors such as hypertension, hyperlipidaemia and diabetes mellitus (affecting 15.6\%, 13.6\% and 6.9\% of the population, respectively). ${ }^{2}$ The Singapore Myocardial Infarction Registry (SMIR) revealed a significant increase in the crude incidence rate of acute myocardial infarction (AMI) from 235.6 to 364.8 per 100,000 population from 2010 to 2019 , with a 30 -day case fatality rate of $8.4 \% .^{3}$

In 1977, Reimer et al. elegantly described using experimental circumflex artery ligation the wavefront phenomenon of myocardial infarction propagation. ${ }^{4}$ They demonstrated that myocardial necrosis started in the subendocardial myocardium after coronary artery occlusion and, with increasing duration of ischaemia, irreversible injury progressed as a wavefront towards the subepicardial myocardium. It was noted that after 40 minutes of occlusion, $55 \%$ of the myocardium at risk was still salvageable by reperfusion; by 3 hours, the salvageable area was down to $33 \%$; and in 6 hours, only $16 \%$. Therefore, any benefit of reperfusion therapy is time-dependent and should be implemented as soon as possible after an acute coronary artery occlusion to delay and prevent ischaemic myocardial cell death. Primary percutaneous coronary intervention (PCI) is now the preferred revascularisation strategy for ST-segment elevation myocardial infarction (STEMI) as it had demonstrated greater reduction in mortality, nonfatal reinfarction and stroke compared to thrombolytic therapy and is currently the standard of care for STEMI in Singapore. ${ }^{5}$

The "total ischaemic time" includes several key time intervals that are determinants of patient outcomes and should be systemically collected and analysed (Fig. 1). They include, temporally, time from symptom onset to first medical contact ("patient delay"), time from first medical contact to arrival at a PCI-capable centre ("emergency medical service [EMS] delay"), and time from arrival at a PCI-capable centre to revascularisation ("in-hospital delay" i.e. the "door-to-balloon" [DTB] time). The optimal approach to reduce each time interval differs in terms of strategy and medical resources needed.

With the recognition that earlier revascularisation confers better survival with less long-term adverse consequences e.g. poor left ventricular function, guidelines from international societies, such as the American College of Cardiology and American Heart Association, have advocated a DTB time of 90 minutes or less, which has become a key performance indicator in many healthcare systems. ${ }^{6}$ DTB time refers to the time from arrival at the Emergency Department (ED) to restoration of antegrade flow down the infarct-related artery (either at the time of balloon inflation or wire crossing of the culprit lesion); it reflects the timeliness of hospitals in treating STEMI patients and quantifies the "in-hospital delay".

Is there a limit beyond which further reduction in the DTB time does not lead to additional improvement in clinical outcomes? Nallamothu et al. performed a multilevel model analysis of the US National Cardiovascular Data Registry (NCDR) CathPCI Registry and showed that every 10 -minute reduction in patient-specific DTB time was strongly and consistently associated with lower in-hospital mortality (adjusted odds ratio [aOR] $0.92,95 \%$ confidence interval [CI] $0.91-0.93, P<0.0001)$ and 6-month mortality (aOR $0.94,95 \%$ CI $0.93-0.95, P<0.0001)$ at the individual patient-level although there was no association between decreases in annual DTB times and mortality at the population-level because of secular trends towards increased mortality risk in the primary PCI population. ${ }^{7}$ More recently, a Taiwan registry demonstrated that patients with a DTB time of less than 60 minutes had lower incidences of thrombolysis in myocardial infarction flow grade less than 3 (aOR $0.4,95 \%$ CI $0.20-0.76$ ), 30-day reinfarction (aOR 0.3,

\footnotetext{
${ }^{1}$ Department of Cardiology, Tan Tock Seng Hospital, Singapore

${ }^{2}$ Department of Emergency Medicine, Tan Tock Seng Hospital, Singapore

Correspondence: A/Prof Yew Woon Chia, Department of Cardiology, Tan Tock Seng Hospital, 11 Jalan Tan Tock Seng, Singapore 308433.

Email: yew_woon_chia@ttsh.com.sg
} 


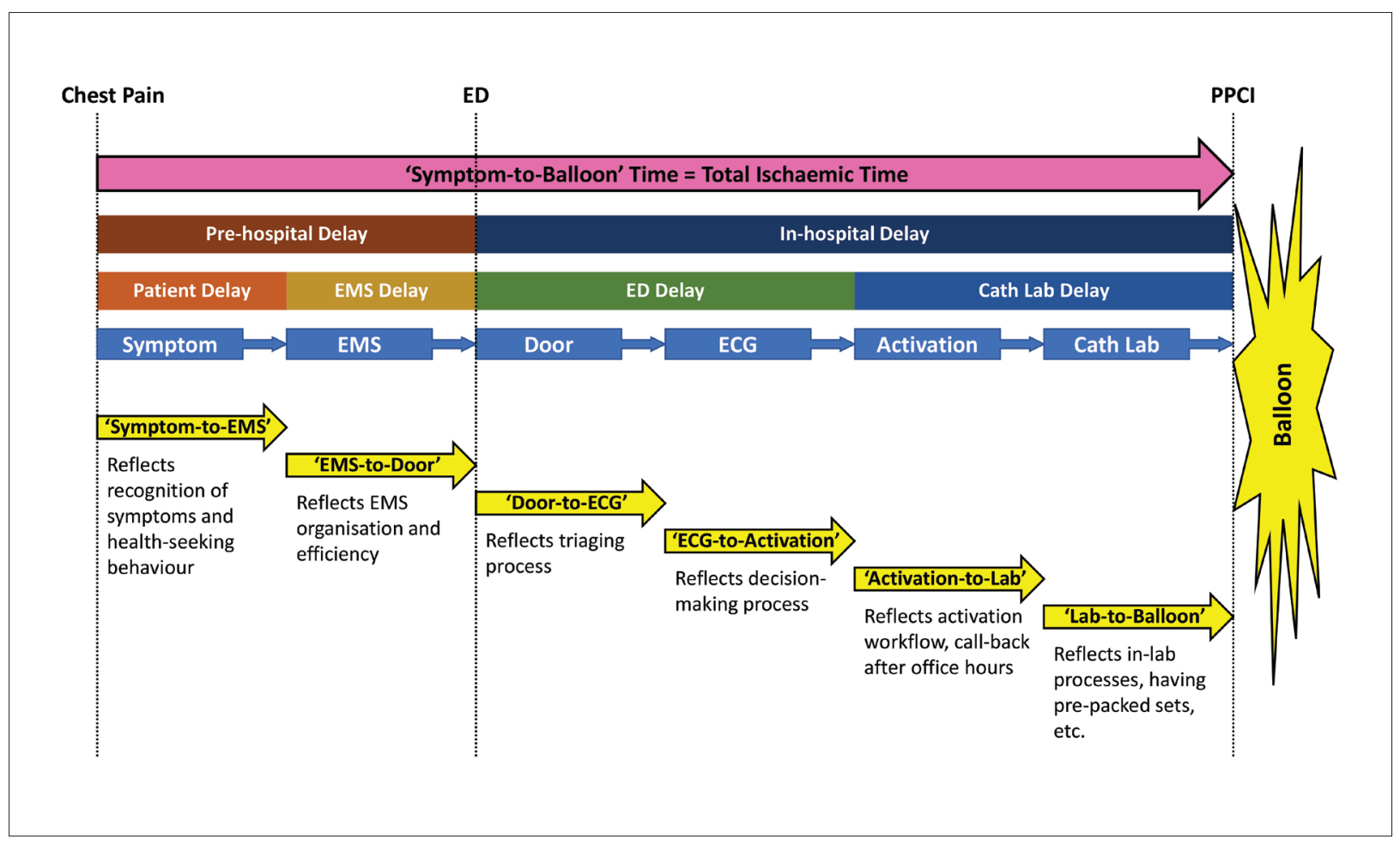

Fig. 1. The "Total Ischaemic Time", time intervals and components.

Cath Lab: cardiac catheterisation laboratory; ECG: electrocardiogram; ED: emergency department; EMS: emergency medical service; PPCI: primary percutaneous coronary intervention

95\% CI 0.10-0.91) and 30-day mortality (aOR 0.3 , 95\% CI 0.09-0.77) than those with a DTB time of 60 to 90 minutes. $^{8}$ Park et al. also revealed, using the Korea Acute Myocardial Infarction Registry, that every 30-minute reduction in the DTB time showed continuous reduction in 1-year mortality (90 to 60 minutes: absolute risk reduction [ARR] 2.4\%, number needed to treat [NNT] $41.9 ; 60$ to 30 minutes: ARR 2.0\%, NNT 49.2). ${ }^{9}$

The SMIR indicated that, barring exceptional cases of equivocal electrocardiographic (ECG) changes or patients who needed prolonged resuscitation or occasions when there were delays in obtaining informed consent, our local healthcare institutions are able to achieve a DTB time of 90 minutes or less in more than $90 \%$ of the time. ${ }^{3}$ An earlier study by Sim et al. identified major system and non-system factors causing delays in the DTB time in a Singapore PCI-capable centre. ${ }^{10}$ Among 1,268 STEMI patients who underwent primary PCI over a 4-year period, 202 (15.9\%) had a DTB time of more than 90 minutes. The most common reasons were a delay in ED processes, atypical clinical presentation and unstable patient condition requiring stabilisation. It was reported that the delayed group had a $2.6 \%$ higher in-hospital mortality, although this did not reach statistical significance.

Initiatives to improve the DTB time have often focused on ED processes (e.g. "door-to-ECG" time, "door-to-cardiac catheterisation laboratory activation" time, "activation-to-arrival at cardiac catheterisation laboratory" time, etc.) and cardiac catheterisation laboratory processes (e.g. activation workflow especially after office hours, logistics such as having prepacked cardiac catheterisation sets, and having a well-rehearsed cardiac catheterisation team). ${ }^{11}$ Improvements in these components have contributed to a significant reduction in the DTB time.

Optimal care of a STEMI patient demands a coordinated, multidisciplinary approach and prehospital processes play an equally significant role. In this issue of the Annals, Liu et al. performed a retrospective cohort study of 321 STEMI patients who presented to the ED of a PCI-capable centre and underwent primary PCI. They compared the DTB time between patients who arrived via emergency ambulances with those who self-conveyed to the hospital. ${ }^{12}$ The investigators showed that the median DTB time was shorter for patients who were transported to the 
hospital via the Singapore Civil Defence Force (SCDF) ambulances (52 minutes, interquartile range [IQR] 45-61) compared to those coming by their own transport (67 minutes, IQR 59-74, $P<0.001$ ). Furthermore, 74 $(74.7 \%)$ patients in the ambulance group achieved a DTB time of less than 60 minutes compared to only $67(30.2 \%)$ patients in the self-conveyance group. Nonetheless, the lack of a mortality benefit despite the shorter DTB time will need further evaluation. It might be because patients who came by the ambulances were sicker as shown in earlier studies. ${ }^{13,14}$

Liu et al. further analysed the components of the DTB time, which were affected by the different modes of transport, and revealed that the improvement in DTB time was attributed to a shortening in the ED processes, such as the door-to-ECG time and the door-to-activation time. This is not entirely surprising because when a STEMI diagnosis is suspected in the prehospital setting, the SCDF ambulance will convey the patient to a PCI-capable centre. The receiving hospital ED will also be notified to standby and the patient's ECG will be transmitted to the ED before ambulance arrival, which had been shown in a previous Singapore before-and-after study to reduce the DTB time by 23 minutes. ${ }^{15}$ The awaiting ED team will also be primed to perform a confirmatory ECG immediately upon patient's arrival and the cardiac catheterisation team may also be activated in advance if the prehospital ECG is clearly diagnostic of a STEMI. Both prehospital ECG transmission and prehospital cardiac catheterisation laboratory activation, which are only possible when patients are conveyed by the emergency ambulances, can clearly contribute to a shorter DTB time.

It was noted that self-conveyed patients had a median door-to-ECG time (11 minutes, IQR 5-15 versus 1 minute, IQR $0-3 ; P<0.001)$ and a median door-to-activation time (18 minutes, IQR $12.0-25.0$ vs 2 minutes, IQR $0.1-7.5 ; P<0.001)$, which were significantly longer compared to patients who arrived via the EMS. This provides an opportunity to explore quality improvement initiatives to reduce the gap and enhance the triaging of walk-in patients who may have symptoms suggestive of an AMI. ${ }^{16}$ In addition, the authors revealed a significantly longer DTB time after office hours attributed to a $21.1 \%(95 \%$ CI $13.2-28.3, P<0.001)$ prolongation in the lab-to-balloon time. Healthcare institutions should collect more information on this and refine the activation workflow outside office hours.

While not specifically studied in the paper, conveyance by ambulance also provides an added layer of safety as AMI patients have a propensity to deteriorate and may go into cardiogenic shock or suffer a cardiac arrest en route to the ED. The patients are closely monitored in the ambulance and the trained paramedics will be able to commence immediate resuscitation, including providing supplementary oxygen, performing defibrillations and administering medications. All these may confer a higher chance of survival compared to patients taking either private or public transport to the hospital.

Unfortunately, only 99 (30.8\%) patients in this study arrived in the ED via the EMS. Although the SMIR indicated an overall higher ambulance-conveyance rate of about $50 \%$, this is still much lower compared to a Canadian registry of $59.9 \% .^{14}$ There is certainly much work for the cardiovascular community to study the health-seeking behaviour of patients, educate the public in recognising the signs and symptoms of an AMI, and call 995 early.

Liu et al. had highlighted through the findings of their study the importance of public education to promote awareness of AMI symptoms and use of the EMS to improve STEMI outcomes. Concurrently, as there will always be self-conveyed patients, we should look at refining the triaging of patients who walk into ED with chest pain syndromes and how activation workflows can be enhanced after office hours.

The Singapore healthcare system has done well to meet the international benchmark DTB time of 90 minutes. We could certainly reduce this further but we should also look into shortening the prehospital delay (Fig. 1). We should collect routine data on "symptomto-balloon" (STB) time as this reflects the total ischaemic time and had been shown to correlate better with infarct size and left ventricular ejection fraction compared to DTB time. ${ }^{17}$ Importantly, the STB time incorporates a patient's dimension into the quality matrix as it includes the time from symptom onset to first medical contact (the "patient delay") and reflects the success of our public educational efforts. The trends of both DTB time and STB time should be monitored in a national STEMI registry with steps taken to identify and address system and non-system related delays.

Ultimately, the overarching goals in treating patients with AMI are to reduce their mortality and improve their left ventricular function. Beyond tracking time metrics such as DTB and STB times, the holistic management of a STEMI patient will also necessarily include compliance with guideline-directed medical therapy, aggressive cardiovascular risk factors control, lifestyle modifications, and participating in a tailored cardiac rehabilitation programme. 


\section{REFERENCES}

1. Principal Causes of Death. Ministry of Health, Singapore. Available at: https://www.moh.gov.sg/resources-statistics/ singapore-health-facts/principal-causes-of-death. Accessed on 6 September 2021

2. National Population Health Survey Report 2019. Health Promotion Board, Singapore. Available at: https://www.hpb.gov.sg/community/ national-population-health-survey/survey-findings. Accessed on 7 September 2021.

3. Singapore Myocardial Infarction Registry Annual Report 2019. National Registry of Diseases Office, Singapore. Available at: https://www.nrdo.gov.sg/publications/ami. Accessed on 6 September 2021.

4. Reimer KA, Lowe JE, Rasmussen MM, et al. The wavefront phenomenon of ischemic cell death. 1. Myocardial infarct size vs duration of coronary occlusion in dogs. Circulation 1977;56:786-94.

5. Keeley EC, Boura JA, Grines CL. Primary angioplasty versus intravenous thrombolytic therapy for acute myocardial infarction: a quantitative review of 23 randomised trials. Lancet 2003; 361:13-20.

6. Antman EM, Anbe DT, Armstrong PW, et al. ACC/AHA guidelines for the management of patients with ST-elevation myocardial infarction; A report of the American College of Cardiology/American Heart Association Task Force on Practice Guidelines (Committee to Revise the 1999 Guidelines for the Management of patients with acute myocardial infarction). J Am Coll Cardiol 2004;44:E1-211.

7. Nallamothu BK, Normand SL, Wang Y, et al. Relation between door-to-balloon times and mortality after primary percutaneous coronary intervention over time: a retrospective study. Lancet 2015;385:1114-22.

8. Chen FC, Lin YR, Kung CT, et al. The Association between Door-to-Balloon Time of Less Than 60 Minutes and Prognosis of Patients Developing ST Segment Elevation Myocardial Infarction and Undergoing Primary Percutaneous Coronary Intervention. Biomed Res Int 2017;2017:1910934.
9. Park J, Choi KH, Lee JM, et al. Prognostic Implications of Door-to-Balloon Time and Onset-to-Door Time on Mortality in Patients With ST-Segment-Elevation Myocardial Infarction Treated With Primary Percutaneous Coronary Intervention. J Am Heart Assoc 2019;8:e012188.

10. Sim WJ, Ang AS, Tan MC, et al. Causes of delay in door-to-balloon time in south-east Asian patients undergoing primary percutaneous coronary intervention. PLoS One 2017;12:e0185186.

11. Krumholz HM, Bradley EH, Nallamothu BK, et al. A campaign to improve the timeliness of primary percutaneous coronary intervention: Door-to-Balloon: An Alliance for Quality. JACC Cardiovasc Interv 2008;1:97-104.

12. Liu Z, Lim MJ, Pek PP, et al. Improved door-to-balloon time for primary percutaneous coronary intervention via emergency ambulance service. Ann Acad Med Singap 2021;50:671-8.

13. Chia YC. Modes of arrival, door to balloon time and its impact on morbidity and mortality for ST elevation myocardial infarct. Crit Care 2012;16(Supp 1):P183.

14. So DY, Ha AC, Turek MA, et al. Comparison of mortality patterns in patients with ST-elevation myocardial infarction arriving by emergency medical services versus self-transport (from the prospective Ottawa Hospital STEMI Registry). Am J Cardiol 2006;97:458-61.

15. Ong ME, Wong AS, Seet CM, et al. Nationwide improvement of door-to-balloon times in patients with acute ST-segment elevation myocardial infarction requiring primary percutaneous coronary intervention with out-of-hospital 12-lead ECG recording and transmission. Ann Emerg Med 2013;61:339-47.

16. Lee CK, Meng SW, Lee MH, et al. The impact of door-toelectrocardiogram time on door-to-balloon time after achieving the guideline-recommended target rate. PLoS One 2019;14:e0222019.

17. Redfors B, Mohebi R, Giustino G, et al. Time Delay, Infarct Size, and Microvascular Obstruction After Primary Percutaneous Coronary Intervention for ST-Segment-Elevation Myocardial Infarction. Circ Cardiovasc Interv 2021;14:e009879. 